\title{
TAGUNG
}

\section{Zur Zukunft der Wirtschafts- und Währungsunion: Integrationsschub wider Willen?}

\author{
Bela Böhme, Manuel Mohr und Julian Plottka*
}

Die Jahreskonferenz des Instituts für Europäische Politik (IEP) in Zusammenarbeit mit dem Wissenschaftlichen Direktorium war den unterschiedlichen Dimensionen der aktuellen Debatte über die Zukunft der Wirtschafts- und Währungsunion gewidmet. Einen Schwerpunkt der Diskussionen bildete das kurz zuvor von der Europäischen Kommission vorgelegte Legislativpaket ${ }^{1}$ mit Vorschlägen zur Reform des Verfahrens bei übermäßigem Defizit und der haushaltspolitischen Überwachung im Euroraum sowie zur Schaffung von Instrumenten zur Vermeidung und Korrektur makroökonomischer Ungleichgewichte.

Diese Vorschläge zur wirtschaftspolitischen Koordinierung wurden dabei in den Kontext der seit Langem insbesondere zwischen Frankreich und Deutschland geführten Debatte über eine europäische ,Wirtschaftsregierung' gestellt. Im Rahmen der Tagung wurde die Bewertung des Legislativpakets und weiterer derzeit diskutierter Reformoptionen durch grundlegendere Fragestellungen nach den Ursachen und den Rahmenbedingungen der Krise ergänzt. Hier wurde das institutionelle Design der Wirtschafts- und Währungsunion ebenso thematisiert wie Motivationen hinter dem Entstehen nationaler Budgetdefizite und Optionen zu ihrer Überwindung. Das bestehende Primärrecht wurde dabei nicht allein auf Notwendigkeiten zur Reform hin untersucht, sondern es wurde auch seine handlungsbegrenzende und -ermöglichende

\section{Die Zukunft der Wirtschafts- und Währungsunion}

Jahreskonferenz des Instituts für Europäische Politik (IEP) in Zusammenarbeit mit dem Wissenschaftlichen Direktorium

\section{Mit freundlicher Unterstützung der Fritz-Thyssen-Stiftung}

Berlin, 7./8. Oktober 2010

\section{Begrüßung}

Matthias PETSCHKE, Leiter der Vertretung der Europäischen Kommission in der Bundesrepublik Deutschland, Berlin

Prof. Dr. Michael KREILE, Vorsitzender des Wissenschaftlichen Direktoriums; HumboldtUniversität zu Berlin

Dr. Elfriede REGELSBERGER, Stellvertretende Direktorin, Institut für Europäische Politik (IEP), Berlin

Die internationale Finanz- und Wirtschaftskrise als Stressfaktor für die Eurozone Vorsitz: Prof. Dr. Michael KREILE, Vorsitzender des Wissenschaftlichen Direktoriums; Humboldt-Universität zu Berlin

\section{Kurzstatements}

Prof. Dr. Ansgar BELKE, Universität DuisburgEssen und Deutsches Institut für Wirtschaftsforschung (DIW), Berlin

Prof. Dr. Rolf CAESAR, Universität Hohenheim, Stuttgart

Prof. Dr. András INOTAI, Generaldirektor, Institut für Weltwirtschaft, Ungarische Akademie der Wissenschaften, Budapest

\footnotetext{
Bela Böhme, Institut für Europäische Politik, Berlin. Manuel Mohr, M.A., Institut für Europäische Politik, Berlin. Julian Plottka, Dipl.-Pol., Institut für Europäische Politik, Berlin.

1 Als Überblick zum Legislativpaket siehe: Europäische Kommission: Wirtschaftspolitische Steuerung in der EU: Kommission legt umfassendes Legislativpaket vor, Dok. IP/10/1199.
} 
Wirkung auf die kommenden Politikentscheidungen diskutiert. Im Zentrum aller Debatten stand dabei die Frage, ob die Krise einen weiteren Integrationsschub auslösen wird.

Die Finanz- und Wirtschaftskrise: Katalysator einer strukturellen Krise?

Das erste Panel der Tagung widmete sich dem Thema: „Die internationale Finanz- und Wirtschaftskrise als Stressfaktor für die Eurozone". Es wurde von der Leitfrage bestimmt, wie sich trotz der Bedingungen der Finanzkrise die Stabilität der Eurozone gewährleisten lässt.

Die Leistungsbilanzunterschiede zwischen den Mitgliedstaaten, die im Zuge der Krise zu Tage getreten sind, wurden als das derzeit dringlichste Problem erkannt. Vor diesem Hintergrund wurden die verschiedenen Möglichkeiten der wirtschaftspolitischen Koordinierung innerhalb Europas in der Fiskal- und Geldpolitik erörtert sowie die fiskalischen Aktivitäten der Europäischen Zentralbank (EZB) bewertet. Es müsste hierbei jedoch die hohe globale Liquidität und die daraus resultierende Abhängigkeit Europas von Akteuren wie den USA und China berücksichtigt werden. Gleichzeitig wurde die Frage aufgeworfen, inwieweit, angesichts des vorherrschenden Misstrauens den regulierenden Kräften der Märkte gegenüber, eine vertiefte politische Union die einzige Alternative sei.

Einigkeit herrschte darüber, dass die Finanzkrise neben dem Problem der drastischen Staatsverschuldungen, das durch die Krise zwar verstärkt wurde, aber dessen Ursachen doch in nationalen Politiken zu suchen seien, vor allem eine Governance-Krise hervorgebracht habe. Zur Lösung dieser sei die Schaffung einer gemeinsamen Wirtschaftspolitik in einer Wirtschafts- und Währungsunion selbstverständlich. In der Debatte sei bisher noch nicht deutlich genug geworden, dass diese nicht zwangsläufig auf eine Zentralisierung und einen anwachsenden EU-Kompetenzkatalog hinauslaufe und auch nicht zu einer Einschränkung der Unabhängigkeit der EZB
Divergenz, Disziplin und Wettbewerbsfähigkeit: die Härtung des Stabilitäts- und Wachstumspaktes

Vorsitz: Prof. Dr. Wim KÖSTERS, Ruhr-Universität Bochum und Rheinisch-Westfälisches Institut für Wirtschaftsforschung (RWI), Essen

\section{Kurzstatements}

Barbara BÖTTCHER, Leiterin Wirtschafts- und Europapolitik, Deutsche Bank Research, Frankfurt am Main

Prof. Dr. Henrik ENDERLEIN, Hertie School of Governance, Berlin

Dr. Reinhard FELKE, Europäische Kommission, GD Wirtschaft und Finanzen, Brüssel

Dr. Martin HEIPERTZ, Bundesministerium der Finanzen, Berlin

Der Ausbau der Wirtschafts- und Währungsunion: das rechtliche Instrumentarium Vorsitz: Prof. Dr. Siegfried MAGIERA, Deutsche Hochschule für Verwaltungswissenschaften Speyer

\section{Kurzstatements}

Prof. Dr. Ulrich HÄDE, Europa-Universität Viadrina, Frankfurt an der Oder

Prof. Dr. Peter-Christian MÜLLER-GRAFF, Universität Heidelberg

Politische Voraussetzungen fiskalischer Disziplin und finanzieller Solidarität in der Eurozone Vorsitz: Prof. Dr. Gianni BONVICINI, Executive Vice President, Istituto Affari Internazionali (IAI), Rom

\section{Kurzstatements}

Dr. Friedrich HEINEMANN, Zentrum für Europäische Wirtschaftsforschung (ZEW), Mannheim Dr. Michael DAUDERSTÄDT, Friedrich-Ebert-

Stiftung, Bonn

Dr. Charalampos KOUTALAKIS, Universität Athen

Institutionelle Lösungen für die europäische ,Wirtschaftsregierung“"

Vorsitz: Prof. Dr. Wolfgang WESSELS, Universität zu Köln

Kurzstatements

Prof. Dr. Heinrich SCHNEIDER, Ehrenvorsitzender des Wissenschaftlichen Direktoriums; Universität Wien

Dr. Arndt FREYTAG VON LORINGHOVEN, Ministerialdirigent, Stellvertretender Leiter der Europaabteilung, Beauftragter für Grundsatzfragen der EU, Auswärtiges Amt, Berlin Prof. Dr. Joachim SCHILD, Universität Trier 
führen müsse. Stattdessen würden die Unterschiede eines französischen und eines deutschen Governance-Modells diskutiert, wodurch die Debatte um eine europäische Wirtschaftsregierung zu europapolitischem Sprengstoff avanciere, wie die Äußerungen von Christine Lagarde gezeigt hätten.

Grundlage für diese Governance-Debatte war die Annahme, die Eurozone an sich sei zwar ein Erfolgsmodell, gleichzeitig seien aber viele der heutigen Probleme als systemimmanent und als Durchsetzungsfehler zu betrachten. Es wurde diskutiert, dass der Euro-Rettungsschirm notwendig gewesen sei, die strukturellen Probleme aber, besonders hinsichtlich seines absehbaren Auslaufens, nicht aus dem Blick verloren werden dürften. Auch werde der Abbau der öffentlichen Verschuldung zunehmend eine Frage von Solidarität und Verantwortung. Die Unterscheidung von Eurozonen- und Nicht-Eurozonenmitgliedern berge die Gefahr einer Zwei-Klassen-Struktur unter den Mitgliedstaaten.

Das Legislativpaket der Kommission: Bewertung im Lichte des aktuellen Reformbedarfs

$\mathrm{Zu}$ Beginn der Sitzung wurde zunächst die Entstehung der Währungsunion skizziert und auf die Schwächen des Stabilitäts- und Wachstumspaktes hingewiesen. Im Lichte der Wirtschafts- und Finanzkrise habe sich das bestehende Modell als nicht funktionsfähig erwiesen und sich die Notwendigkeit grundlegender Änderungen gezeigt. Die Reform des Stabilitäts- und Wachstumspaktes sowie deren mögliche Gestaltung waren vor diesem Hintergrund Gegenstand der Diskussion.

Als Ausgangspunkt für die weitere Debatte wurden die wesentlichen Inhalte des von der Europäischen Kommission jüngst vorgelegten Legislativpakets dargelegt. Obwohl Schwachstellen der Wirtschafts- und Währungsunion seit Längerem bekannt gewesen seien, habe erst die Notwendigkeit der finanziellen Unterstützung Griechenlands die Dringlichkeit einer Reform der bestehenden währungspoliti-

\section{Schlussfolgerungen}

Prof. Dr. Michael KREILE, Vorsitzender des Wissenschaftlichen Direktoriums; HumboldtUniversität zu Berlin

schen Mechanismen der Europäischen Union verdeutlicht. Die neuen Kommissionsvorschläge zielten darauf, zeitnah konkrete Lösungen zu finden, ohne primärrechtliche Änderungen notwendig zu machen. Inhaltlich sei das Legislativpaket zweigeteilt: Es enthalte einerseits Instrumente zur Überwachung der nationalen Haushaltsführung, andererseits zur makroökonomischen Überwachung der Eurozonenmitglieder.

Die von der Kommission vorgeschlagene Stärkung der präventiven Mechanismen und Sanktionsmöglichkeiten sowie die Vorgaben zur nationalen Haushaltsführung wurden unterschiedlich, jedoch überwiegend positiv bewertet. Nichtsdestotrotz wurde selbst von Befürwortern des Legislativpakets angemahnt, dass eine Bewertung nationaler Haushaltslagen neben den öffentlichen Finanzen auch die private Verschuldung mit einbeziehen müsse. In Anbetracht der strukturellen wirtschaftlichen Divergenzen innerhalb der Eurozone wurde jedoch auch generelle Kritik an den Gesetzesvorhaben laut. Die langfristige Stabilität der Eurozone setze nämlich sowohl eine tatsächliche fiskalpolitische Integration als auch eine Vollendung der wirtschaftspolitischen Koordinierung der Mitgliedstaaten voraus beides sei in den Initiativen nicht vorgesehen.

Des Weiteren bestand Einigkeit darüber, dass der Ausschluss und Wiedereintritt einzelner Mitgliedstaaten aus der Eurozone keine gangbare Lösung sei, da dies schwer kalkulierbare systemische Risiken berge. Als Alternativen wurden deshalb die Möglichkeit einer, bailout-Option" sowie die eines Insolvenzverfahrens für Defizitsünder kontrovers diskutiert.

Welche politischen Reformen unter den gegenwärtigen wirtschaftlichen und fiskalischen Umständen umsetzbar seien, wurde im Rah- 
men des Panels ebenfalls thematisiert. In diesem Zusammenhang stand die grundsätzliche Zustimmung der Bundesregierung $\mathrm{zu}$ den Kommissionsvorschlägen sowie deren Präferenz für primärrechtliche Änderungen zur Stärkung des Stabilitäts- und Wachstumspaktes im Mittelpunkt. Widerstand anderer Mitgliedstaaten im Hinblick auf eine erneute Vertragsreform sei zu erwarten, die Differenzen jedoch überbrückbar. Insgesamt müssten neue Mechanismen eingeführt werden, die den bestehenden „European Financial Stability Facility“ (EFSF) ersetzten, um dessen Perpetuierung zu vermeiden.

Die Reform der Wirtschafts- und Währungsunion: rechtliche Grenzen und Chancen

Das dritte Panel war dem rechtlichen Instrumentarium zum Ausbau der Wirtschafts- und Währungsunion gewidmet. Diese verfüge bereits über Instrumente, die daraufhin untersucht werden müssten, ob überhaupt die Notwendigkeit zur Schaffung eines neuen rechtlichen Instrumentariums bestehe. Damit verbunden sei die Frage nach der Eignung des geltenden materiellen Rechts einerseits und des geltenden Verfahrensrechts andererseits. Diesen Fragen wandten sich die Panelisten unter besonderer Berücksichtigung des Legislativpakets der Europäischen Kommission zu.

Auf einer grundsätzlichen Ebene wurde das wechselseitige Verhältnis von Politik und Recht thematisiert. Recht sei zuerst als Ausdruck einer politischen Entscheidung zu einem bestimmten Zeitpunkt zu begreifen. In diesem Sinne stelle das Recht ein Tableau für zu ergreifende Maßnahmen bereit. Zum Zweiten konditioniere Recht die Politik aber auch. Die derzeit diskutierten Reformvorschläge fänden bestehendes Recht und eine funktionierende Union vor. Einige Reformoptionen könnten entsprechend durch Sekundärrechtsakte, andere nur durch Vertragsänderungen umgesetzt werden. Ergänzend bestehe die Möglichkeit, Maßnahmen außerhalb des EURechts zu ergreifen. Drittens schaffe die Verbindung von Recht und Politik Orientierungs- sicherheit. So hätten die geltenden Regeln die Entstehung der Krise nicht verhindert und auch das Primärrecht weise Stellen auf, in denen es nicht eindeutig sei. Dennoch habe die Politik in der Krise auf dieser Basis handeln können.

Diese Erwägungen zur Beziehung von Politik und Recht fanden ihre konkreten Beispiele in der Diskussion der derzeit zur Debatte stehenden Reformoptionen. Die Rechtsgrundlage des jüngsten Legislativpakets, Art. 136 i.V.m. Art. 121 Abs. 6 des Vertrages über die Arbeitsweise der Europäischen Union (AEUV), ermögliche eine verstärkte Koordinierung der Haushalts- und Wirtschaftspolitik in der Eurozone. Hinsichtlich der Zulässigkeit, auf diese Basis die vorgesehene Umkehrung der Beschlussfassung im Rat zu einem befristeten Ablehnungsrecht zu stützen, wurden jedoch deutliche Zweifel geäußert.

Genereller wurden die Optionen zur Schaffung eines permanenten Beistandsmechanismus diskutiert. Art. 122 Abs. 2 AEUV lasse nur Notstandsmaßnahmen zu. Als Rechtsgrundlage für einen permanenten Beistandsmechanismus komme deshalb allein Art. 352 AEUV infrage. Die Fortentwicklung des Primärrechts mithilfe dieser Flexibilitätsklausel stehe aber gemäß $\S 8$ des Integrationsverantwortungsgesetzes unter dem Vorbehalt einer Zustimmung des Deutschen Bundestages.

\section{Mangelhafte Haushaltsdisziplin: Gründe und Auswege}

Als politische Voraussetzungen wurden hemmende und förderliche Faktoren für die fiskalische Disziplin von Staaten sowie Optionen zum Abbau von Staatsschulden diskutiert. Zudem gewährte das vierte Panel Einblicke in die laufende griechische Debatte.

Warum sind Haushaltsdefizite so populär? Unter der Prämisse des Bestehens demokratischer Entscheidungsverfahren wurde die Präferenzstruktur der Wähler zur Beantwortung der überspitzten Ausgangsfrage herangezogen. Diese tendierten dazu, kurzfristige indi- 
viduelle Vorteile den langfristigen gesamtgesellschaftlichen Folgen von Verschuldung vorzuziehen. Die These von der Wirkung der parteiideologischen Färbung der Regierung auf das Verschuldungsniveau finde hingegen keinen ausreichenden empirischen Beleg. Ein stark polarisierter Parteienwettbewerb habe aber diese Wirkung. Daneben wurde der Einfluss institutioneller Faktoren untersucht. So sei gut belegbar, dass föderal organisierte Staaten zu einer höheren Verschuldung tendierten. Ein Faktor, der Staatsdefizite begrenze, seien verfassungsrechtliche Verschuldungsgrenzen.

Nach einem Blick auf die Rahmenbedingungen der Entstehung von Verschuldung folgte eine Erörterung der Optionen zum Abbau von Staatsschulden. Dazu sei ein neuer, nicht mehr eindimensionaler Blick auf Schulden notwendig: Schulden eines Akteurs seien die Investitionen eines anderen und generierten für diesen Wohlstand. Vor diesem Hintergrund böten sich drei Optionen an, Schulden zu überwinden: 1. Die Durchführung einer Umschuldung, die aber derzeit die Gefahr einer neuen Finanzkrise berge, da die Gläubiger Verluste zu realisieren hätten. 2. Der Schuldner spare, was jedoch dessen wirtschaftliche Entwicklungsperspektive verschlechtere. 3. Die Gläubiger stärkten die Wirtschaft der Schuldner durch eine Steigerung ihrer Importe aus den Schuldnerstaaten. So bestünde zwar die Gefahr einer Inflation, es werde aber Wachstum generiert. Deshalb seien eine Überwachung der Leistungsbilanzsalden sowie eine Orientierung der Fiskalpolitik an einer dynamischen statt einer statischen Schuldengrenze zu begrüßen.

Ergänzt wurde die theoretische Diskussion um Einblicke in die griechische Debatte als Rahmenbedingung für das weitere Handeln des Landes zur Bewältigung der Krise. Dort sei die Auffassung verbreitet, dass das Land die Verantwortung habe, die eigenen Probleme zu lösen und die notwendigen Maßnahmen zu ergreifen. Die Krise werde als Chance begriffen, um seit Langem notwendige Refor- men nun umzusetzen, was bereits begonnen habe. Dass die Union nahezu unvorbereitet von der Krise getroffen worden sei, zeige die Notwendigkeit, auf EU-Ebene ein Instrumentarium zu schaffen, um künftig schneller auf Krisen reagieren zu können. Gerade, dass das jüngste Legislativpaket der Kommission eine Diskussion über die unausgewogenen Außenhandelsbilanzen angestoßen habe, wurde aus griechischer Sicht begrüßt.

\section{Die ,Europäische Wirtschaftsregierung':} Facetten eines schillernden Begriffs

Es wurde zunächst dargelegt, dass die Idee einer Wirtschaftsregierung nicht neu ist, wie die Geschichte der Initiativen zeigt, die bis zu den späten 1960er Jahren und der Einrichtung der Zollunion zurückreiche. Stets seien die Diskussionen von einem internen Tauziehen und der Dominanz nationalstaatlicher Interessen bestimmt worden. Daraus resultierend seien bindende Regeln, die zu einer vertieften wirtschaftlichen Integration geführt hätten, immer ausgehebelt worden. Ob eine Wirtschaftsregierung heute in Form einer Institution jenseits von Kommission und Rat sowie nationalstaatlichen Partikularinteressen möglich sei, hänge nicht zuletzt auch von der Einsicht der Bürgerinnen und Bürger ab.

Daran anschließend wurde deutlich, dass eine effizientere wirtschaftspolitische Koordinierung notwendig ist. Diese müsse sich, im Gegensatz zu dem Symptome bekämpfenden Euro-Rettungsschirm, um die Ursachen kümmern. Nicht nur die Überwachung von Haushaltsdefiziten, sondern auch die bis zum Inkrafttreten Vertrages von Lissabon noch geltenden Regelungen des Maastrichter Vertrages hätten sich hinsichtlich einer langfristigen Koordinierung von Strukturpolitiken als unzureichend erwiesen. Ein regelbasiertes und weniger politisiertes System, das unter Setzung der politischen Rahmenbedingungen die Wettbewerbsfähigkeit ins Zentrum stellt, sei notwendig. Einig wurde man sich auch darüber, dass die Hauptanpassungslast bei den Defizitländern liege, aber auch Überschuss- 
länder wie Deutschland ihrer Mitverantwortung nachkommen müssten.

Ein weiterer Schwerpunkt der Diskussion lag auf den Missverständnissen im französischdeutschen Dialog um den , schillernden ' Begriff einer Wirtschaftsregierung. Hier wurde deutlich, dass die französischen und deutschen Positionen letztendlich doch nicht allzu weit auseinander liegen. Beide Staaten hätten Interesse an durchsetzungsfähigen Regeln fiskalpolitischer Disziplin, einer optimierten Abstimmung von Fiskal- und Geldpolitik sowie einer Koordinierung der nationalen Fiskalpolitiken. Konsens herrschte darüber, dass die Diskussion auf eine mögliche Integration von Strukturpolitiken, ein Instrument zur Bewertung der Wettbewerbsfähigkeit, Fragen zur Steuerung der Wechselkurspolitik und eine Nutzung des EU-Budgets als Mittel zur makroökonomischen Stabilisierung ausgeweitet werden muss.

\section{Europa 2010: Vertiefung in der Not?}

Abschließend war ein eher verhaltener Ausblick der Tagung auf die deutsche Europapolitik in der Krise zu konstatieren. So unterliege die deutsche Europapolitik in den Reformverhandlungen einer Reihe von Restriktionen, die die Durchsetzung deutscher Positionen nicht erleichterten. Es gebe Inkonsistenzen in der deutschen Position, der innerstaatliche Konsensbedarf sei durch das
Bundesverfassungsgericht erhöht worden und die große Zahl der Mitgliedstaaten stelle auch größere Anforderungen an die Konsensfindung.

In der deutschen Haltung zur Korrektur makroökonomischer Ungleichgewichte wurde ein Wandel der Debatte konstatiert. Zuerst habe ein gewisser ,Anti-Lagarde-Effekt ‘ vorgeherrscht, während jüngst selbst der deutsche Bundeswirtschaftsminister Rainer Brüderle für höhere Löhne plädiere. Letztlich seien in der Frage ausgewogener Außenhandelsbilanzen die Anpassungslasten asymmetrisch verteilt. Europaweit habe Deutschland als Referenzpunkt mitgliedstaatlicher Reformbemühungen an Bedeutung gewonnen, diese seien jedoch dadurch begrenzt, dass nicht alle Nationalökonomien exportgetrieben sein könnten.

Am Ende der Tagung wurde die eingangs formulierte Frage klar beantwortet: Es sei in allen Panels deutlich geworden, dass der durch die Wirtschafts- und Finanzkrise entstandene Sanierungsbedarf einen Integrationsschub ausgelöst habe. Allerdings handele es sich dabei um einen Integrationsschub wider Willen, an dem sich manche Mitgliedstaaten nur aus der Not heraus beteiligten. Eine Verfassungsrevolution sei dagegen nicht zu erwarten. Der Ausschluss oder der Austritt von Mitgliedstaaten aus der Eurozone sei dagegen keine Lösung. 